\title{
A nonlinear method of removing harmonic noise in geophysical data
}

\author{
Y. Jeng and C.-S. Chen \\ Department of Earth Sciences, National Taiwan Normal University, 88, Sec. 4, Ting-Chou Road, Taipei, 116, Taiwan
}

Received: 3 March 2011 - Revised: 22 April 2011 - Accepted: 2 June 2011 - Published: 21 June 2011

\begin{abstract}
A nonlinear, adaptive method to remove the harmonic noise that commonly resides in geophysical data is proposed in this study. This filtering method is based on the ensemble empirical mode decomposition algorithm in conjunction with the logarithmic transform. We present a synthetic model study to investigate the capability of signal reconstruction from the decomposed data, and compare the results with those derived from other 2-D adaptive filters. Applications to the real seismic data acquired by using an ocean bottom seismograph and to a shot gather of the ground penetrating radar demonstrate the robustness of this method. Our work proposes a concept that instead of Fourier-based approaches, the harmonic noise removal in geophysical data can be achieved effectively by using an alternative nonlinear adaptive data analysis method, which has been applied extensively in other scientific studies.
\end{abstract}

\section{Introduction}

The interference of harmonic noise in geophysical data has long been a nuisance problem for geophysicists. The noise commonly originates from power-line harmonic radiation, global lightning, EM transmitters, oscillating sources, and inadequate data processing (Butler and Russell, 1993; Jeng et al., 2007; Bagaini, 2010). It may contaminate the data directly during time-domain measurements of EM fields, or indirectly, by EM coupling to instrument cables during the data acquisition and perhaps by artifacts produced during the data processing (Butler and Russell, 2003; Huang and Wu, 2008). Various methods have been proposed to suppress harmonic noise in geophysical records. Conventional Fourier based linear filtering techniques are not desirable mainly due to the

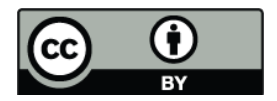

Correspondence to: Y. Jeng (geofv001@ntnu.edu.tw) results of signal distortions and artifacts generated. Subtracting an estimate of the harmonic noise from the recorded data is the current main approach (Nyman and Gaiser, 1983; Butler and Russell, 1993; Jeffryes, 2002; Meunier and Bianchi, 2002; Butler and Russell, 2003; Saucier et al., 2006). The estimate includes the estimating of harmonic noise fundamental frequency, and amplitudes and phases of all harmonics. However, most of the spectrum estimation related methods pointed out above are critically relied on the accuracy of the estimated fundamental frequency; normally the allowed error is less than $0.02 \%$. Furthermore, these methods are derived under the assumption that each sinusoidal contaminant is stationary, i.e., constant in amplitude, phase, and frequency over the length of the record (Butler and Russell, 2003). This assumption is impractical because the attributes of harmonic noise always drift with time for many reasons. Other effective methods are by using inversion techniques or by implementing filters with pattern-based scheme (Guitton and Symes, 2003; Guitton, 2005; Haines et al., 2007). The inversion technique-based methods require a sufficient number of traces, and are applicable if data quality is good. Filters employing pattern models are effective but they are time-consuming, and adequate pattern models are necessary for filter estimation (Haines et al., 2007).

This research is motivated by interest in designing a nonlinear, adaptive filtering scheme derived from the EEMD (ensemble empirical mode decomposition) method originally proposed by Wu and Huang (2005). Through a series of nonstationary adaptive sifting processes based on the local characteristics of the data, this method decomposes a set of data into components that have physical significance with meaningful instantaneous time-frequency description. In view of this, the proposed method could be used to the study of harmonic noise removal in geophysical data because the harmonic noise patterns are easy to identify as long as they are not mixed with the signal. Another desired function of this filter is the feasibility of dealing with non-stationary data

Published by Copernicus Publications on behalf of the European Geosciences Union and the American Geophysical Union. 
without the need of accuracy constrain as required in conventional spectrum estimation approaches. To make the method applicable to geophysical data which always suffer from severe attenuation, we modify the procedure by adding the logarithmic transform to the data before decomposition. In this paper, we use a synthetic model analysis and field data acquired from an ocean bottom seismograph (OBS) and from the ground penetrating radar (GPR) to demonstrate how the proposed method can be utilized as an effective tool in harmonic noise removal.

\section{Methods}

This section briefly describes the technique applied to remove the harmonic noise that affects geophysical records. More details can be found in the contributions of Huang et al. (1998) and Wu and Huang (2005, 2009). The adaptive data analysis technique that we adopted includes the empirical mode decomposition (EMD) algorithm with its revision EEMD method. In this study, we generally call this new and evolving technique "the EEMD method" which includes the original EMD algorithm and the Hilbert-Huang Transform (HHT). The application of this analysis has been widely demonstrated in the recent peer-reviewed literature both in science and engineering communities, and it has been verified very effective in spectrum analysis and noise filtering (Gloerson and Huang, 2003; Flandrin et al., 2004; Peel and McMahon, 2006; Battista et al., 2007; Jeng et al., 2007; Huang and Wu, 2008; Feldman, 2009; Tsolis and Xenos, 2009; Jackson and Mound, 2010; Lee and Ouarda, 2010; Lin and Jeng, 2010). Some contributions of the EMD technique have been made on the seismic data processing; however, due to the high attenuation nature of the reflection data, it still has room for more elaborate studies. In this paper, we propose a modified scheme which is useful in applying the EEMD method to remove the harmonic noise in reflection data.

Our hypothesis of utilizing this technique in removing the harmonic noise is that a set of recorded data can be considered a gather of many simple oscillatory modes of different frequencies, and we can localize an event in both time and frequency domains by the proposed decomposition procedure. Huang et al. (1998) defined the simple oscillatory mode as the intrinsic mode function (IMF) which is a function satisfying two conditions: (1) the number of extrema and the number of zero-crossings must either equal or differ at most by one; and (2) at any point, the mean value of the envelope determined by the local maxima (upper envelope) and the envelope determined by the local minima (lower envelope) is zero. As a result, each decomposed component, the IMF, has instantaneous frequency with physical meaning, and the harmonic noise should be separated from the signal in accordance. Our previous work has investigated various cases using the EMD method, suggesting that the separation of different intrinsic oscillatory modes of the reflection data is feasible. (a)
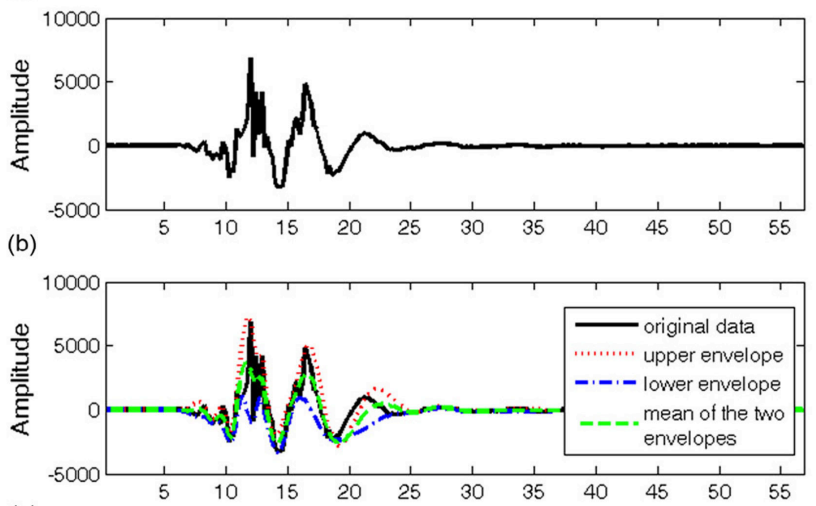

(c)

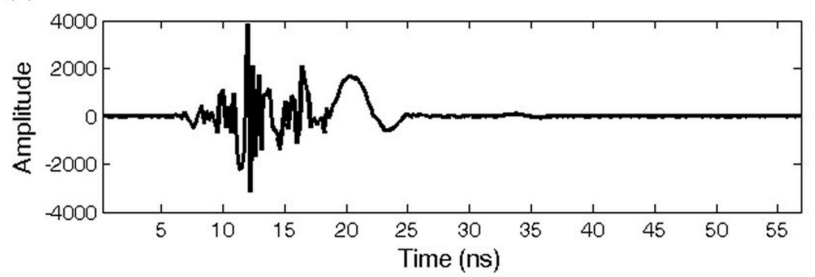

Fig. 1. Process of sifting. (a) The original single trace data $g(t)$ extracted from a typical GPR record acquired in the field. (b) Original data $g(t)$ with upper and lower envelopes and the mean $m_{1}$ of the two envelopes. (c) The first mode, $d_{1}$, which is the difference between the original data and $m_{1}$. This is not an IMF yet.

The EMD algorithm is briefly summarized as follows by using a typical GPR single trace, $g(t)$, acquired in the field. To begin with, we establish the upper and lower envelopes for this given data set $g(t)$ (Fig. 1a, b) and compute the mean of the two envelopes $m_{1}$ (Fig. 1b). It follows that the difference between the input data $g(t)$ and $m_{1}$ is the prototype of the first mode (Fig. 1c), $d_{1}$, in prototype:

$d_{1}=g(t)-m_{1}$

This operation is called "sifting", which is for the purpose of removing background waves of the IMF and of making the data profiles more symmetric. If $d_{1}$ doesn't satisfy the conditions of IMF, the sifting process must be repeated until an IMF is obtained. Theoretically, the sifting process could be carried on to the extreme but the resulting IMF may approach a stationary mono-frequency component as derived from the Fourier decomposition. Therefore, a stoppage criterion is required to ensure that the resulting IMFs (IMF components) retain useful information. As proposed by Huang and $\mathrm{Wu}$ (2008), a global stoppage can be accomplished by a Cauchy type of convergence test, which indicates that for the given data set with $T+1$ samples, the size of the standard deviation after $j$ times of sifting 


$$
\mathrm{SD}_{j}=\frac{\sum_{t=0}^{T}\left|d_{j-1}(t)-d_{j}(t)\right|^{2}}{\sum_{t=0}^{T} d_{j-1}^{2}(t)}
$$

should be smaller than a predetermined value (values between 0.2 and 0.3 are suggested). Another simpler but maybe better criterion is that the sifting process stops only the output consistently (stay the same for between 3 and 8 consecutive times of sifting) fulfills the definition of IMF (Huang et al., 1998; Huang and $\mathrm{Wu}, 2008)$. If $d_{1 j}\left(d_{1}\right.$ mode function after $j$ times sifting) satisfies the stoppage criterion, then we define it as $c_{1}$, which is the first IMF of the given data set $g(t)$ with the highest frequency. The component $c_{1}$ can be subtracted from the original data $g(t)$ to obtain a residue $r_{1}$ which still contains lower frequency components. The residue $r_{1}$ is then treated as the new data ready for the next sifting process. The original data are therefore iteratively decomposed into $n$ IMFs $\left(c_{1}\right.$ to $c_{n}$ ) of different frequency band and a residue $r_{n}$ is obtained after repeating the sifting procedure $n$ times as

$g(t)=\sum_{i=1}^{n} c_{i}+r_{n}$

A more robust sifting algorithm, EEMD, was proposed by Wu and Huang $(2005,2009)$ as a revision of the EMD. This new version adopts the noise-assisted data analysis approach (NADA) and noise-assisted signal extraction (NASE) technique into EMD algorithm to eliminate the mode mixing problem of EMD which occurs as a consequence of signal intermittency. The EEMD method can be illustrated by simple mathematical expressions as follows. Before sifting process, we add finite amplitude white noise $w(t)$ to the input data $g(t)$ to get the noise added data $G(t)$ as

$G(t)=g(t)+w(t) \times R$,

where $R$ is the ratio of the standard deviation of the added noise amplitude to that of the original data $g(t)$. The EMD is then applied to $G(t)$ to generate the noise added IMFs. By repeating $k$ times of the noise adding procedure with different white noise series but of the same amplitude each time, an ensemble of the corresponding $i$ th IMF component, $E_{i}$, will be:

$E_{i}(t)=\sum_{j=1}^{k} c_{i j}(t)$,

where $c_{i j}$ is the $j$ th noise added trial of the $i$ th IMF component of $G(t)$. The mean of the IMFs in ensemble $E_{i}$ is equal to

$\bar{c}_{i}=\frac{1}{k} \sum_{j=1}^{k} c_{i j}$.
As the number of the ensemble member $k$ approaches infinity, the process of taking the mean will cancel out the white noise added on each time of trial due to the randomness of the noise, and the mean, $\bar{c}_{i}$, will approach to the truth of the $i$ th IMF component. Thus, the mode mixing problem of EMD would be greatly alleviated because the added white noises of different scales would make uniformly distributed reference scales available in the whole time-frequency space for the signal to populate, but would cause no interference in the signal.

The actual value of the added noise amplitude $R$ and the number of the ensemble member $k$ are case dependent. Wu and Huang $(2005,2009)$ suggested that 0.2 and a value of few hundreds for the two parameters would lead to a good result in most cases. Lin and Jeng (2010) allowed 0.5 and 100 for $R$ and $k$, respectively, to process their relatively small quantity of data dominated by low frequency signals. In our case, the data quantities are large and most of the data contain high frequency signals; therefore, we use 0.1 for the added noise amplitude $R$, and use 50 for the number of $k$ in EEMD algorithm to compromise with the computer time.

It is important to note that in the procedure of decomposition, the attenuation of reflection data also is a factor of causing the mode mixing problem. The weak later part of a given attenuated signal becomes significant after the prior IMF is subtracted from the original data. Therefore, the attenuated signal may allocate in pieces in more than one IMF. In order to provide a certain solution for signal/noise separation techniques based on the EMD method, we performed the logarithmic transform technique in this study to balance the display of data values and to increase the sensitivity of the decomposition. The advantage of doing this is that the logarithmic transform allows displaying data of large dynamic range without introducing any artificial distortion. Conventionally, some approaches are used to deal with the data attenuation. Among them, the AGC (auto gain control) correction and the energy compensation by using a gain function are two common techniques. The AGC correction is easy to apply, but the original attributes of the data will be distorted after the amplitude adjustment, and further signal analysis could be irrelevant. As for the gain correction, the true inverse $Q$ (attenuating factor) filter for the data cannot be obtained without the help of a precise laboratory measurement or other joint estimates (Irving and Knight, 2003).

To implement an adaptive filter using the EMD or EEMD techniques, the interpreter should have fundamental concept of the signal and noise in the data to be processed, and make basic assumptions for selecting components (Jeng et al., 2007). In general, the selected components must demonstrate convincing physical significance for reconstructing the data. Fortunately, the patterns of signal and noise of reflection data are easy to identify; therefore, we can determine the components for reconstructing the data without difficulties. In case the components contain both signal and noise, further decompositions or a comparison of the reconstruction 
(a)

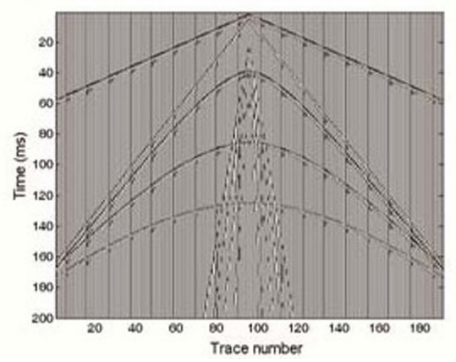

(b)

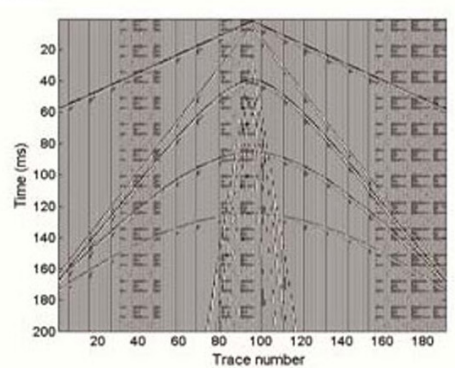

(c)

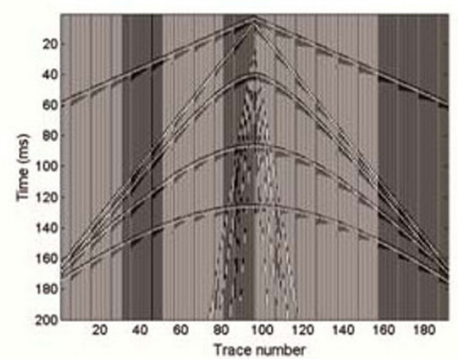

Fig. 2. Simulation of the reflection model. (a) Shot gather model without harmonic noise. (b) Model with harmonic noise and ground roll added. (c) Logarithmic transform performed on the model shown in (b).
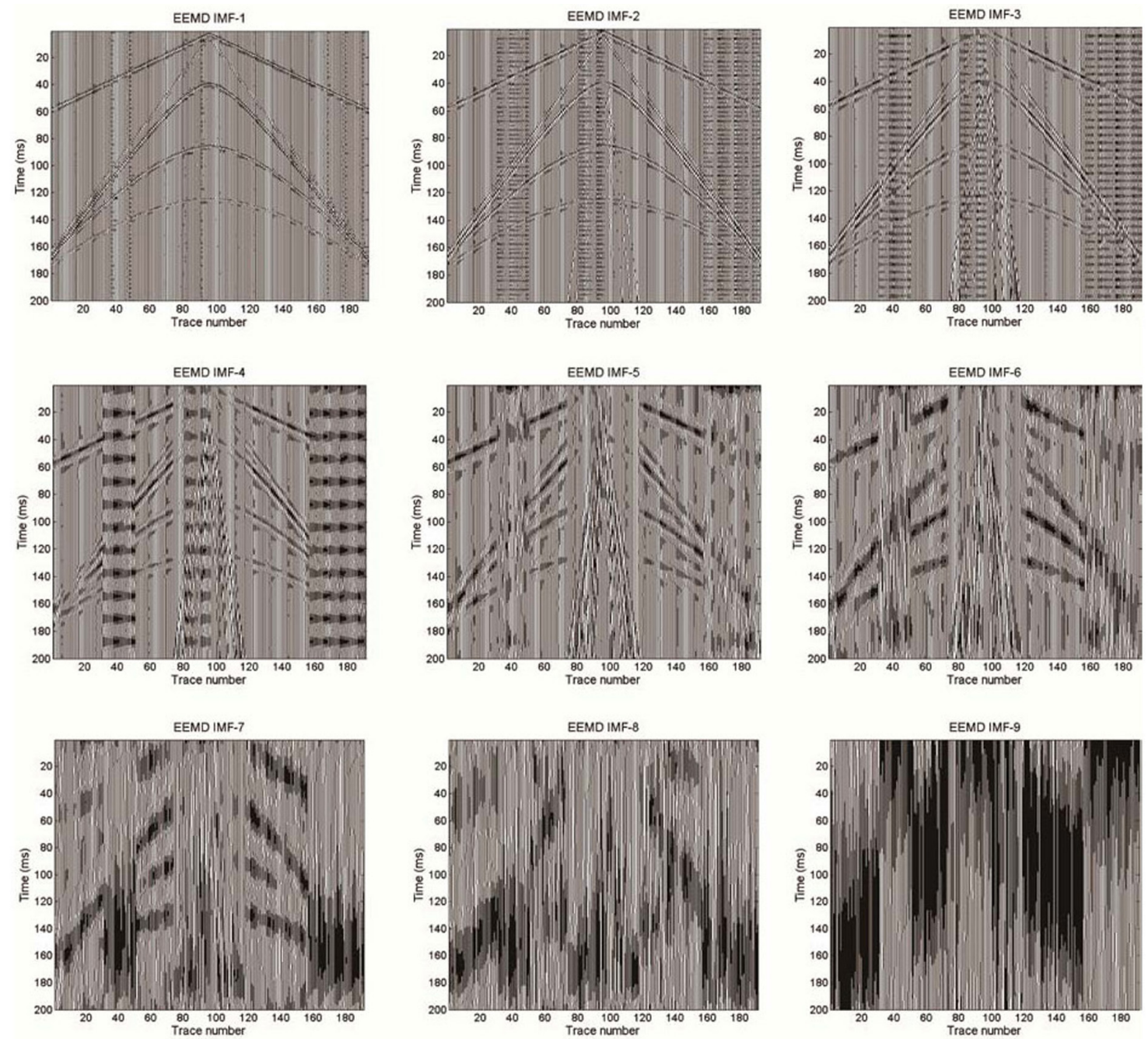

Fig. 3. Filter bank of the harmonic noise affected model data before logarithmic transform. 

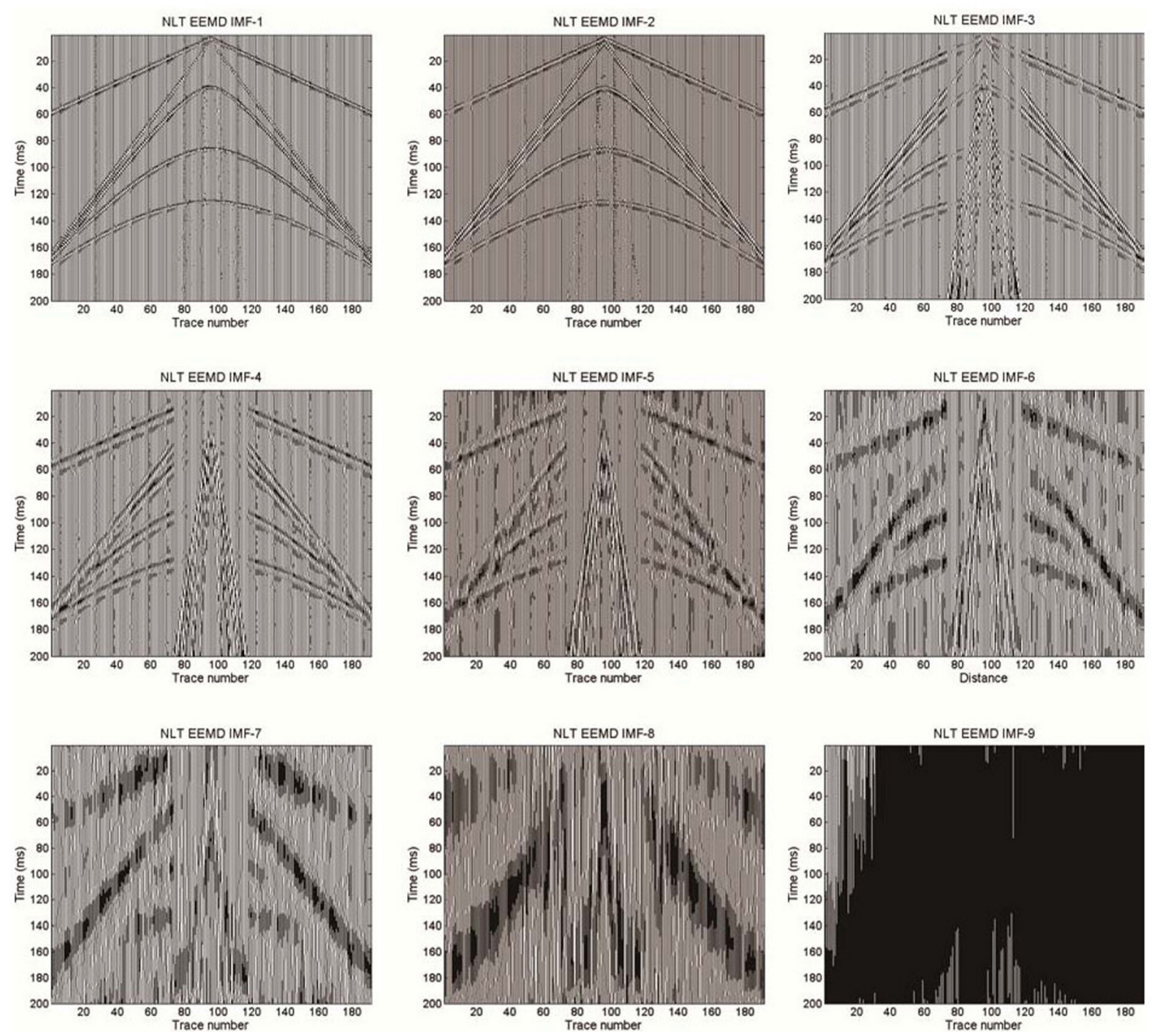

Fig. 4. Filter bank of the harmonic noise affected model data with logarithmic transform.

results using different combinations of components should be done for making an optimal decision. More details of selecting process are illustrated in the synthetic model analysis followed.

The images we treated in this study are collections of single one-dimensional traces. Since the data attributes are one-dimensional, we decompose each trace using onedimensional EEMD method and collect the components of each trace of the same level to construct the two-dimensional image components of each level. From the point of view of image processing, a two-dimensional EMD algorithm should be a good alternative for image decomposition. However, it is not considered in this study due to the reasons of much longer processing time and marginal improved images may not be worth considerable additional endeavors. Another reason is that artifacts may be created by using two-dimensional algorithm to process data of one-dimensional attributes.

\section{Synthetic model analysis}

We consider a simple reflected common-shot gather model contaminated with harmonics. Figure 2 a shows the model without harmonic noise, and the reflection events are at $40 \mathrm{~ms}, 88 \mathrm{~ms}$, and $128 \mathrm{~ms}$. Airwave, direct wave and ground roll are added to make the model look more realistic. The source wavelet used to simulate the signal waveform is a minimum phase impulsive source. Harmonic noise as a sum of $60 \mathrm{~Hz}$ (fundamental frequency), $180 \mathrm{~Hz}$, and $300 \mathrm{~Hz}$ sinusoids is added to the model in three zones as illustrated in Fig. 2b. The processing scheme was implemented by employing the logarithmic transform to the data at first (Fig. 2c), and then by applying the EEMD method to establish a dyadic filter bank (Wu and Huang, 2009; Lin and Jeng, 2010) which contains the decomposed components (IMFs and a residue) of physical significance. Figure 3 shows the dyadic filter bank of nine IMFs derived from the harmonic noise affected synthetic model before logarithmic transform, in which IMF- 
(a)

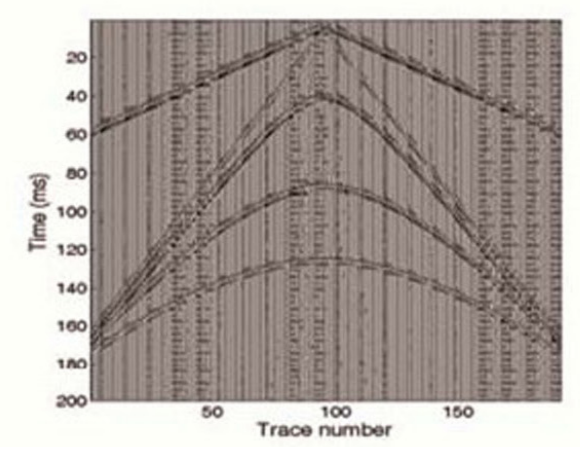

(c)

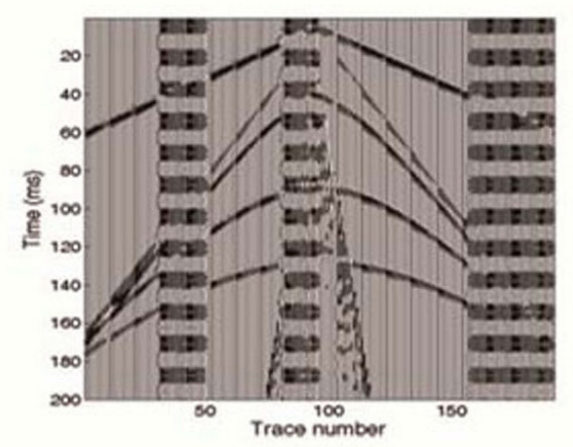

(b)

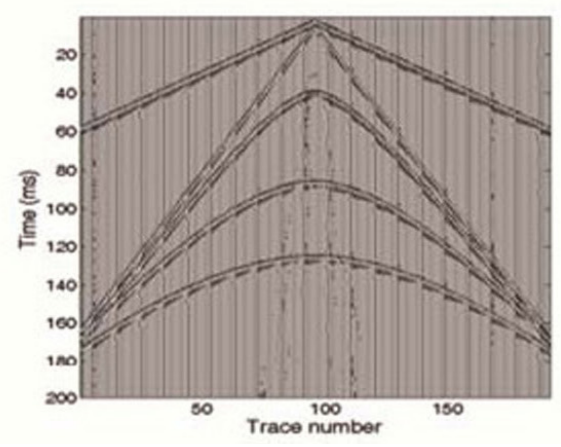

(d)

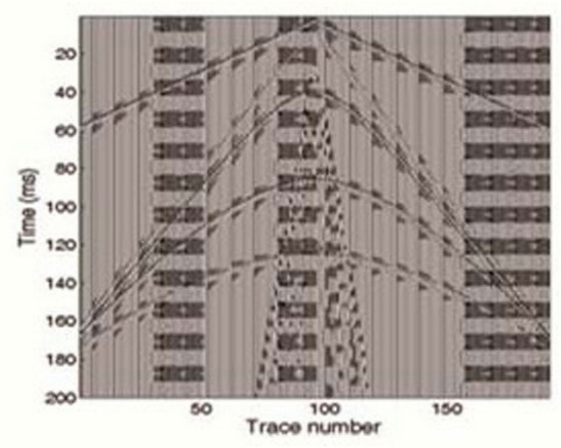

(e)

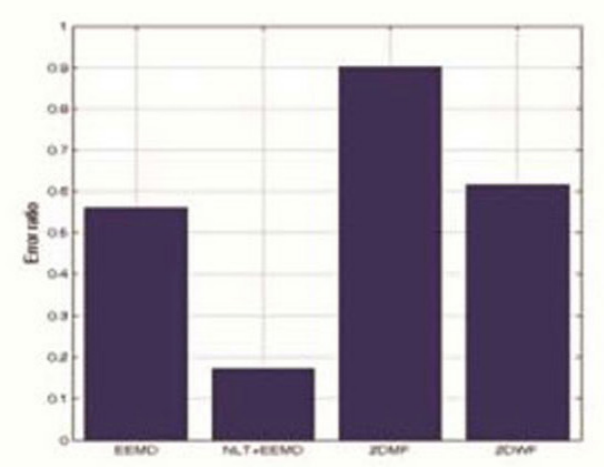

Fig. 5. Comparison of filtering results of the proposed method and other 2-D adaptive techniques to the model contaminated with harmonic noise and ground roll. The amplitudes are shown on the logarithmic scale for comparison. (a) EEMD filtering without the aid of logarithmic transform. Components IMF-2 to IMF-9 were subtracted from the dyadic filter bank to suppress the noise. (b) Logarithmic transform performed before EEMD filtering. Components IMF-3 to IMF-9 were subtracted from the dyadic filter bank in this case. (c) Result of applying the optimum 2-D median filter. (d) Result of applying the 2-D adaptive Wiener filter. (e) Error ratios of the EEMD filter, logarithmic EEMD filter, optimum 2-D median filter (2DMF), and 2-D adaptive Wiener filter (2DWF).

9 is the residue representing the trend or the background energy of the data analyzed. Because the signal and the noise patterns of reflection data are easy to distinguish, the attributes of IMF-1 to IMF-8 in the dyadic filter bank are summarized as follows: IM-1 shows highest $\mathrm{S} / \mathrm{N}$ ratio; most of the $300 \mathrm{~Hz}$ harmonic noise resides in IMF-2; IMF-3 and IMF-4 contain lower harmonic noises and some high frequency ground roll; IMF-5 is clearly affected by ground roll of middle frequency band; IMF-6 to IMF-8 have the low frequency contents of both signal and noise energy. As pointed 
out by $\mathrm{Wu}$ and Huang $(2005,2009)$, the EEMD components of data are not necessarily IMFs, for EEMD involves numerous summations of IMFs. We found that this drawback is more serious when highly attenuated data are analyzed. This is probably due to the dramatic dynamic range change in the sifting process for highly attenuated data, and the coverage of envelopes varies significantly in each mode during the process. Therefore, for EEMD components containing both signal and noise may be treated as raw data and decomposed further to extract more signal out.

However, decomposing the IMFs may increase the decomposition level, increasing simultaneously the possibility of having IMFs which lack physical meaning. In such case, the resulting IMF may approach a stationary mono-frequency component as derived from the Fourier decomposition. The logarithmic transform we used in this study already relaxes the dynamic range problem and improves the sensitivity of the EMD method. Therefore, as for the purpose of recognizing reflection events, more refined models may not indicate better solutions.

The dyadic filter bank of the model with logarithmic transform is demonstrated in Fig. 4. We can see that the sifting process of the EEMD technique worked more precisely in this case. IMF-1 and IMF-2 are dominated by reflection and refraction signal components; IMF-3 to IMF-8 contain both signal and noise of various frequency ranges. Because the logarithmic transform suppressed much of the harmonic energy in this case, it is almost invisible in all the decomposed components.

To proceed with the filtering process, we subtracted the components dominated by harmonic noise and ground roll from the dyadic filter bank, and reconstructed the model using the signal components left. As expected, the logarithmic transform reduced the mode mixing problem, resulting in a better resolution in signal and noise recognition. Figure 5 shows a comparison of the results of EEMD filtering without and with the application of logarithmic transform, and compares them with the results of applying two other adaptive filtering techniques proposed by Jeng et al., 2009. To make the comparison more convincing, the amplitudes of all the figures are presented on the logarithmic scale. Comparing Fig. 5a with Fig. 5b, we found that with the logarithmic transform, less IMFs were subtracted from the data but a better result was achieved. In addition, the suppression of ground roll is an extra credit of using our method; however, the investigator is free to decide which noise to eliminate because most of the harmonic noise and ground roll reside in different components. Figure $5 \mathrm{c}, \mathrm{d}$ indicate the filtering results of the optimum 2-D median filter with $3 \times 15$ window and the 2-D adaptive Wiener filter with $3 \times 17$ window, respectively. A quantitative comparison was made by calculating the error ratios of different filtering processes. The error ratio Err is defined as

Err $=1-$ Corr where Corr indicates the correlation coefficient between the original model without noise added and the filtered result. In our case, we treated the model data $\mathbf{M}$ as an $m \times n$ matrix, and $\mathbf{F}$ represents the filtered data of the same dimension, then

Corr $=\frac{\sum_{m} \sum_{n}\left(\mathbf{M}_{m n}-\overline{\mathbf{M}}\right)\left(\mathbf{F}_{m n}-\overline{\mathbf{F}}\right)}{\sqrt{\left(\sum_{m} \sum_{n}\left(\mathbf{M}_{m n}-\overline{\mathbf{M}}\right)^{2}\right)\left(\sum_{m} \sum_{n}\left(\mathbf{F}_{m n}-\overline{\mathbf{F}}\right)^{2}\right)}}$

where $\overline{\mathbf{M}}$ and $\overline{\mathbf{F}}$ are the means of matrixes $\mathbf{M}$ and $\mathbf{F}$, respectively.

Comparing the filtered results and the original model without noise, the proposed EEMD method with logarithmic transform gives the lowest error ratio (Fig. 5e).

\section{Field data examples}

We present two examples of the practical application of our processing technique. In these real examples, data were processed with and without logarithmic transform for comparison. Figure 6a shows the refraction and reflection data acquired by one of the National Taiwan Ocean University OBSs deployed offshore southwestern Taiwan where is an area very likely deposited with gas hydrate. Two coupled air guns with 275 and 150 cube inches were used as the seismic source, and the shot interval was about $25 \mathrm{~m}$ (Tsai, 2007). The direct wave from the air guns and the reflection from the subsurface of ocean bottom were received by a hydrophone attached to the outside of the OBS case and a geophone array deployed on the ocean bottom, respectively. Probably due to energy transmitted from the electronic and radio devices of the OBS, the data were badly affected by the harmonic noise (Fig. 6a). After logarithmic transform, the resolution of original data is improved (Fig. 6b). A decomposition procedure as executed in the model study was applied to the data. We first decomposed the original data, which resulted in a dyadic filter bank of nine IMF components as shown in Fig. 7. By contrast, the dyadic filter bank derived from the data with logarithmic transform is demonstrated in Fig. 8. Following the selection principle previously given, we eliminated the noise components and reconstructed the data using signal components to remove the harmonic noise. Fig. 9a shows the filtering result of subtracting components dominated by harmonic noise (IMF-4 to IMF-9 in this case) from the data of Fig. 7. For comparison purposes, Fig. 9b demonstrates the result of applying the same procedure to the original data with logarithmic transform. Under this condition, components of IMF-6 to IMF-9 in Fig. 8 were resolved as noise components and eliminated to suppress the harmonic noise. Both results successfully demonstrate the removal of harmonic noise in the OBS data but the improvement of $\mathrm{S} / \mathrm{N}$ ratio of the data with logarithmic transform is more significant. The OBS field data example confirms the feasibility of applying this harmonic noise removal technique to real data. 
(a)

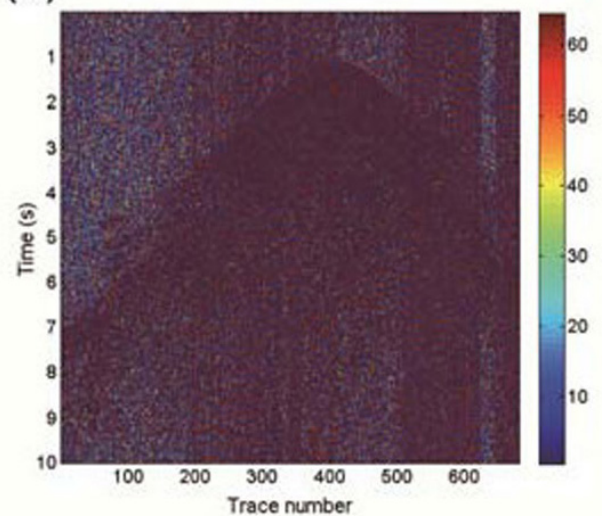

(b)

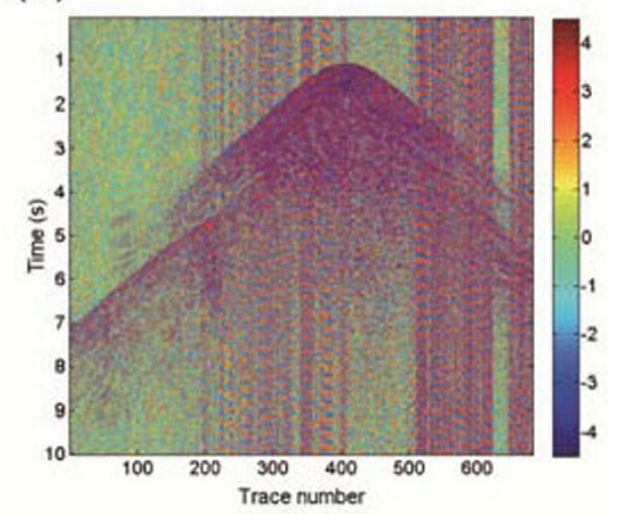

Fig. 6. OBS field data. (a) Original OBS data showing refraction and reflection events and harmonic noise. (b) Logarithmic transform of original OBS data.
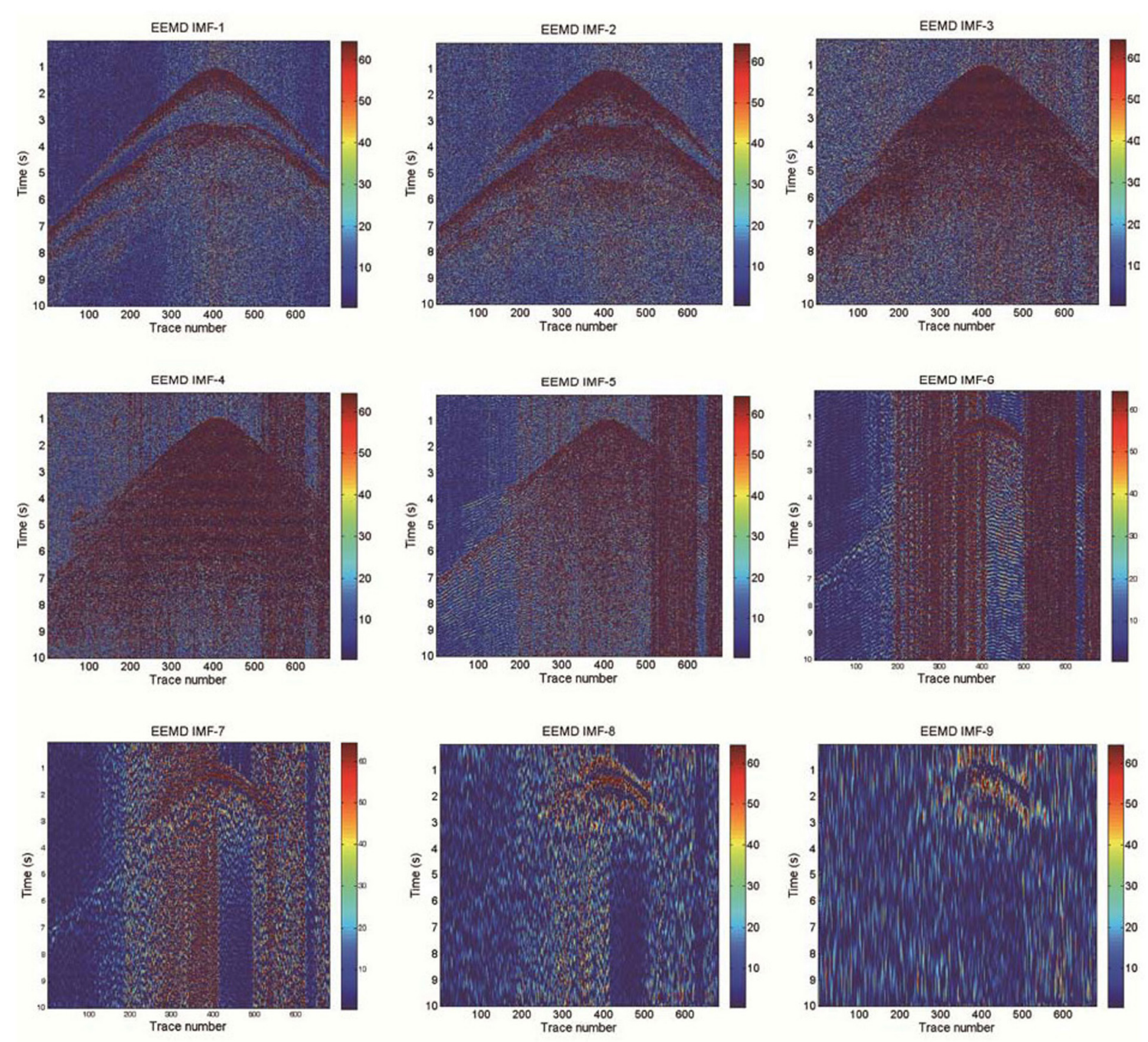

Fig. 7. Filter bank of the original OBS data. 

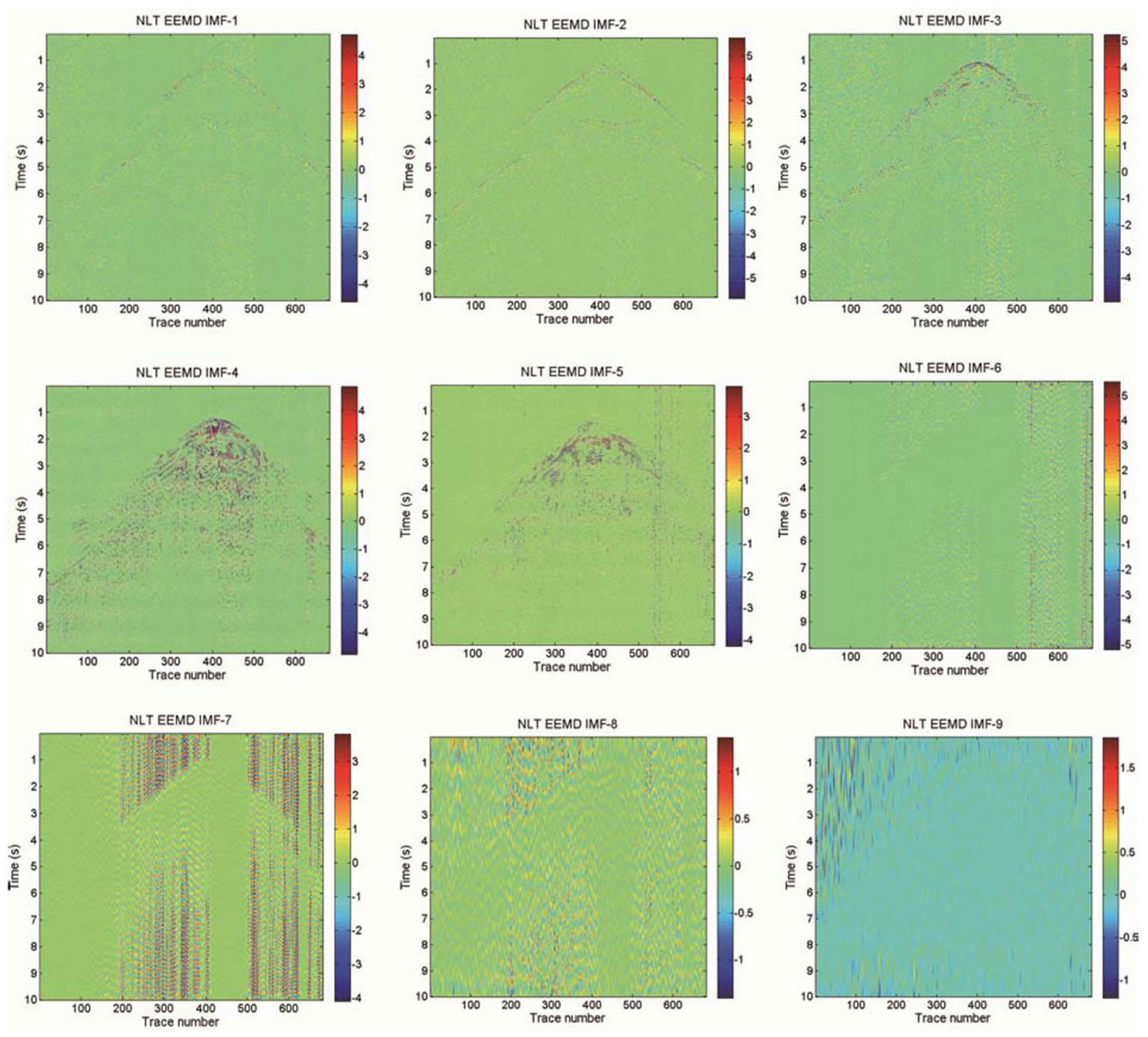

Fig. 8. Filter bank of the OBS data with logarithmic transform.

(a)

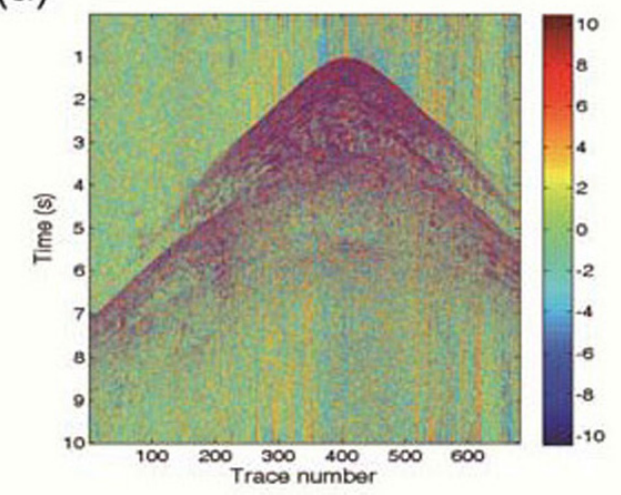

(b)

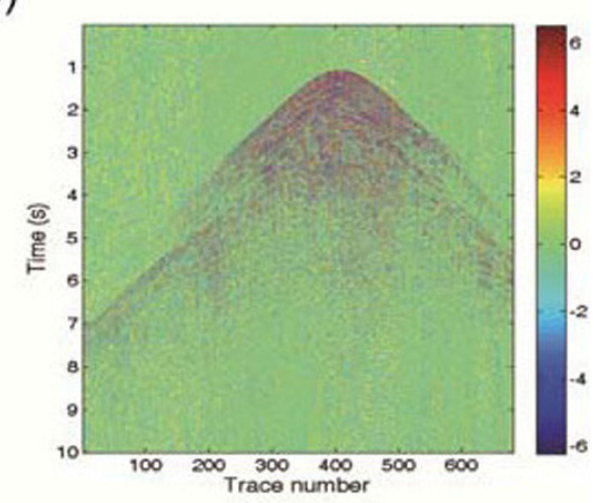

Fig. 9. OBS field data after harmonic noise removal. (a) Result of applying the harmonic noise removing method without the aid of logarithmic transform. Components IMF-4 to IMF-9 were determined as noise and eliminated. (b) Result of applying the harmonic noise removing method to data with logarithmic transform in which components IMF-6 to IMF-9 were determined as noise and eliminated. The amplitudes shown in both figures are on the logarithmic scale. 
(a)

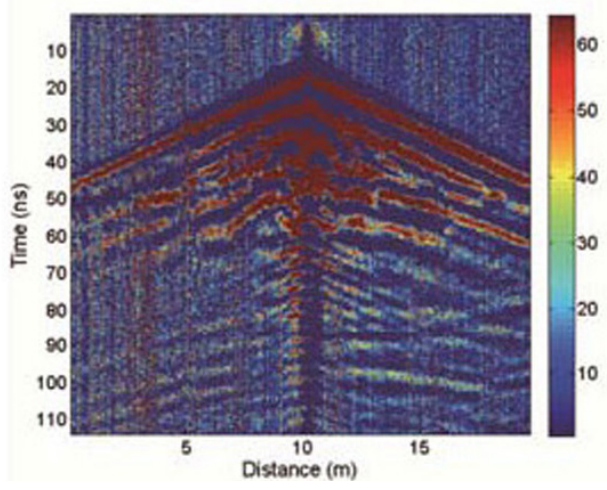

(b)

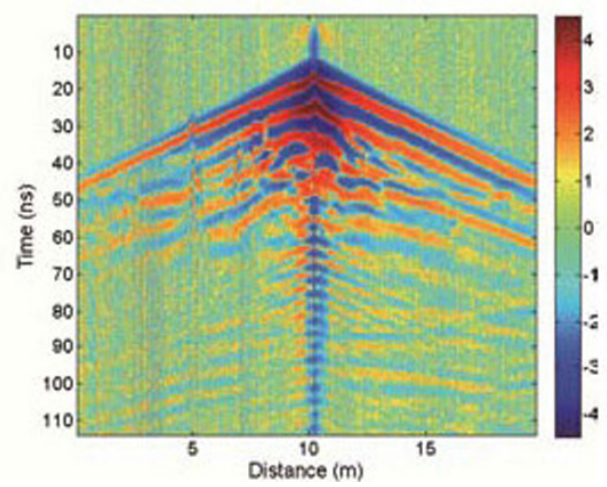

Fig. 10. GPR shot gather data. (a) Original GPR data showing refraction and reflection events and harmonic noise. (b) Logarithmic transform of original GPR data.
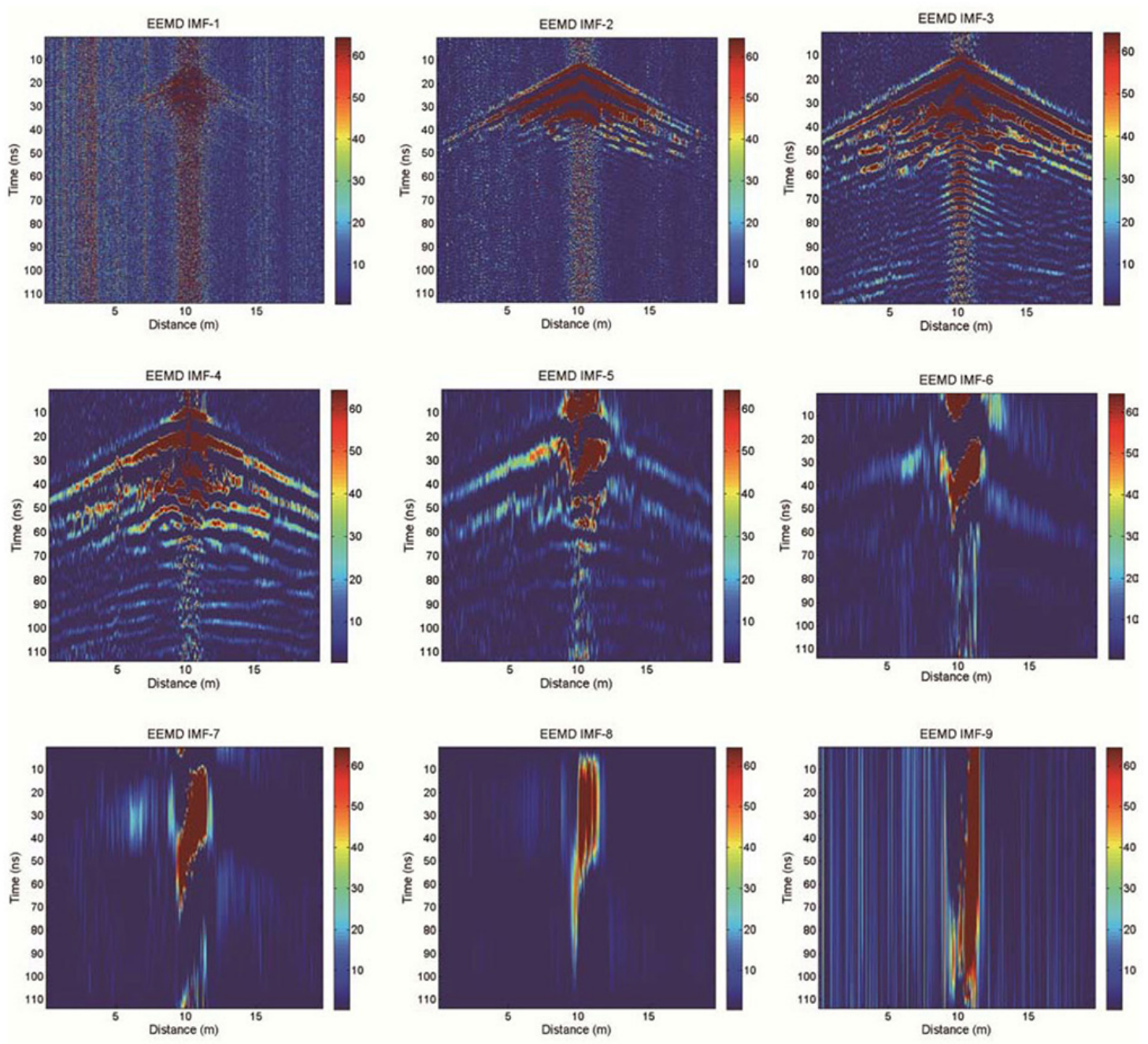

Fig. 11. Filter bank of the original GPR shot gather data. 

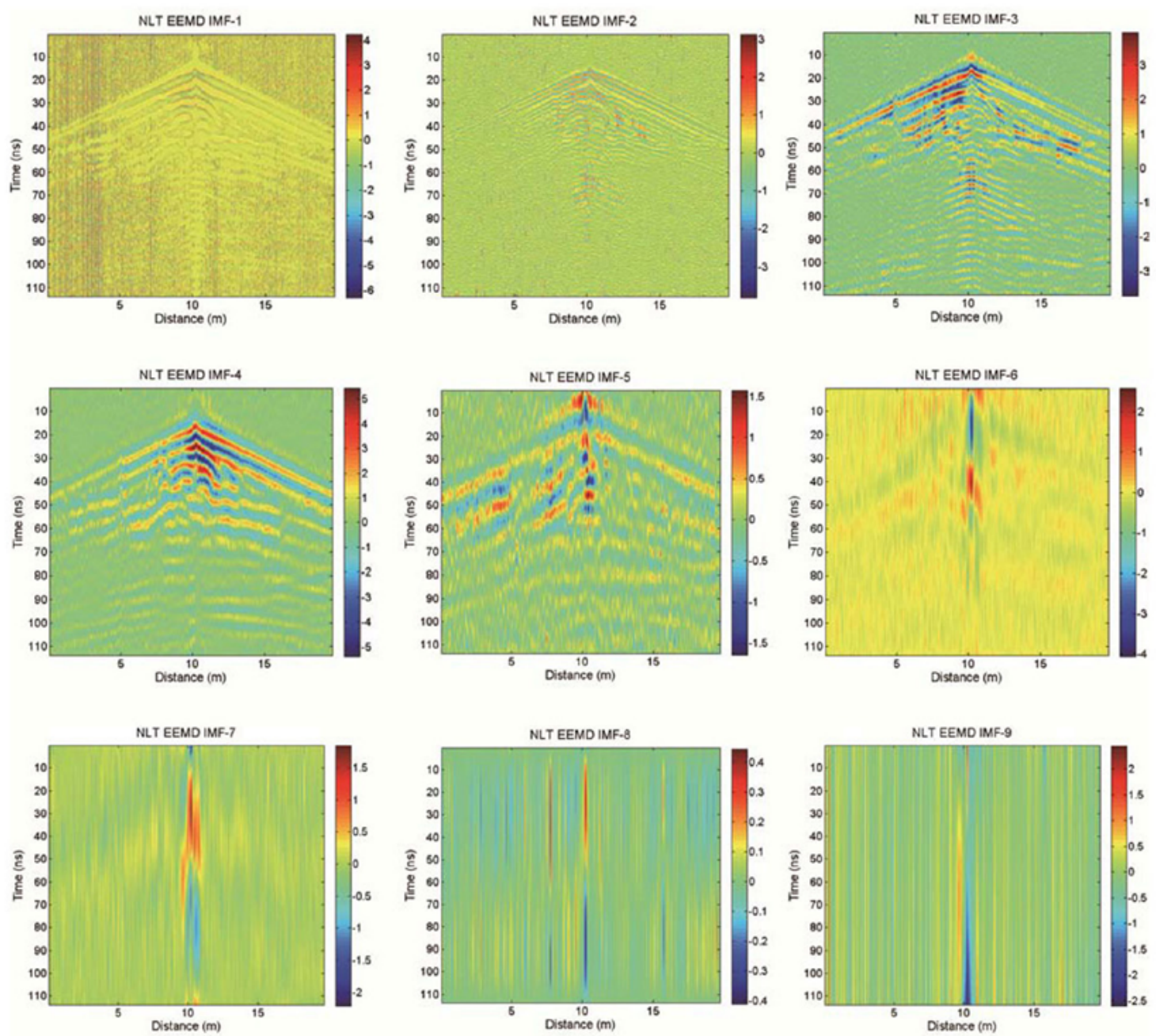

Fig. 12. Filter bank of the GPR shot gather data with logarithmic transform.

(a)

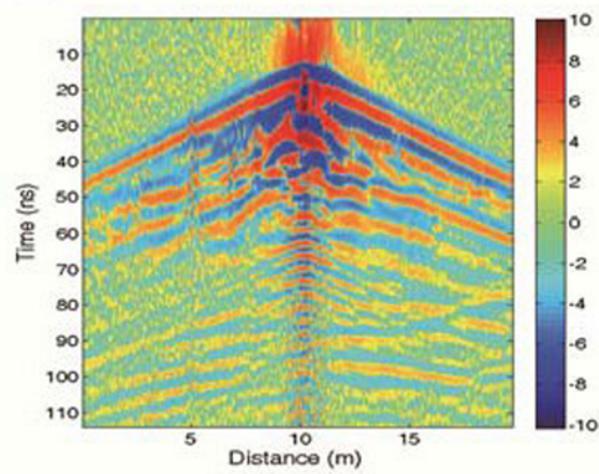

(b)

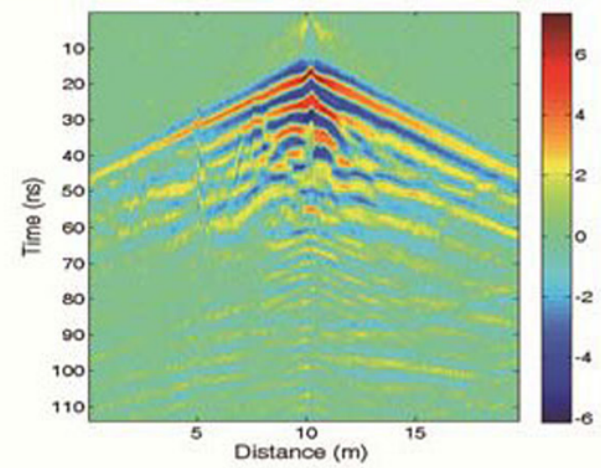

Fig. 13. GPR shot gather data after harmonic noise removal. (a) Result of applying the harmonic noise removing method without the aid of logarithmic transform. Components IMF-3 to IMF-5 were selected for reconstructing the data. (b) Result of applying the harmonic noise removing method to data with logarithmic transform data in which components IMF-3 to IMF-5 were selected for reconstructing the data. The amplitudes shown in both figures are on the logarithmic scale. 
As an example of the versatility of our processing scheme, we applied the proposed method to GPR data. The data were acquired from a GPR survey line traversing a resurfaced road pavement. To carry out the survey, a MALA ProEx radar system with unshielded $200 \mathrm{MHz}$ central frequency antennas was employed. With the transmitter located at the central position (10 $\mathrm{m}$ on the distance axis), the receiving antenna was then moving through the survey line with $5 \mathrm{~cm}$ interval per trace when a split-spread common shot gather was gained. The recording parameters were $114 \mathrm{~ns}$ sampling period with 512 samples (which was equal to about $0.2 \mathrm{~ns}$ sample interval) and 6 vertical stacks per trace. Although the GPR system we employed in this study are equipped with fiber optic cables to minimize the interference of the EM noise, harmonic noise affected traces are still visible in the data - chiefly at the first half of the survey line which is near a building (Fig. 10a). Because the minimum allowable separation distance for $200 \mathrm{MHz}$ antennas is $0.5 \mathrm{~m}$ and our spatial sample interval is $5 \mathrm{~cm}$, the traces near the location of the transmitting antenna (the central region of the shot gather) display unusual high amplitude. This is due to the signal saturation when two antennas are in close proximity to each other. After logarithmic transform, the resolution of the data is improved in general (Fig. 10b). We then decomposed both sets of data using the EEMD technique to establish dyadic filter banks (Figs. 11 and 12). By applying the same selecting principles noted previously, we eliminated components of low $\mathrm{S} / \mathrm{N}$ ratio and reconstructed the data using the signal components. Results are shown in Fig. 13a and b in which the data processed by logarithmic transform (Fig. 13b) demonstrate much better resolution; particularly the signal saturation around the center of the shot gather is greatly reduced in addition to the removal of harmonic noise.

\section{Conclusions}

This study demonstrates a nonlinear and potentially valuable technique in harmonic noise removal. To verify this promising method, we have examined the validation of the proposed processing procedure through the synthetic model study followed by the real data investigations. The results show the robustness of this method. While it is successfully applied to the OBS and GPR reflection data, based on our studies in other fields of geophysics, we believe that the application of this method is not limited to reflection data.

The EMD method has been empirically tested widely in various applications other than geophysical research, giving better results than most of the traditional analysis methods. More importantly, the decomposed components are nonlinear and reveal true physical meanings. With the aid of logarithmic transform, the EEMD method is significantly improved in resolving signal and noise, and is more reliable in processing data with serious attenuation. However, the proposed method is only empirical, and a theoretical foundation should be useful in making unified justifications on the validity of the results deduced. Compared with the previously published work, our method is straightforward in implementation; furthermore, results of the numerical modeling and field examples suggest that the suppression of other noises in addition to the harmonic noise removal is also possible with this method.

Acknowledgements. The authors acknowledge the partly financial support provided by the National Sciences Council of Taiwan, ROC under Grant No. NSC 99-2116-M-003-008. We also thank the OBS team of the Institute of Applied Geosciences, National Taiwan Ocean University for kindly providing the original OBS data and graduate student Pei-Hsin Chen of the Department of Earth Sciences, National Taiwan Normal University for her assistance in collecting the important information of field data. Special thanks go to Luciano Telesca (the editor), Xavier Garcia and one anonymous reviewer for their interest in this work and constructive comments. Finally, we thank G. F. Margrave for sharing the MATLAB function source code of a minimum phase wavelet for impulsive sources.

Edited by: L. Telesca

Reviewed by: X. Garcia and another anonymous referee

\section{References}

Bagaini, C.: Acquisition and processing of simultaneous vibroseis data, Geophysical prospecting, 58(1), 81-99, 2010.

Battista, B. M., Knapp, C., McGee, T., and Goebel, V.: Application of the empirical mode decomposition and Hilbert-Huang transform to seismic reflection data, Geophysics, 72(2), H29-H37, 2007.

Butler, K. E. and Russell, R. D.: Subtraction of powerline harmonics from geophysical records, Geophysics, 58(6), 898-903, 1993.

Butler, K. E. and Russell, R. D.: Cancellation of multiple harmonic noise series in geophysical records, Geophysics, 68(3), 10831090, 2003.

Feldman, M.: Analytical basics of the EMD: Two harmonics decomposition, Mech. Syst. Signal Pr., 23(7), 2059-2071, 2009.

Flandrin, P., Rilling, G., and Gonçalvés, P.: Empirical mode decomposition as a filter bank, IEEE Signal Proc. Lett., 11(2), 112-114, 2004.

Gloerson, P. and Huang, N. E.: Comparison of interannual intrinsic modes in hemispheric sea ice covers and others geophysical parameters, IEEE T. Geosci. Remote Sens., 41(5), 1062-1074, 2003.

Guitton, A.: Multiple attenuation in complex geology with a pattern-based approach, Geophysics, 70(4), V97-V107, 2005.

Guitton, A. and Symes, W.: Robust inversion of seismic data using the Huber norm, Geophysics, 68(4), 1310-1319, 2003.

Haines, S., Guitton, A., and Biondi, B.: Seismoelectric data processing for surface surveys of shallow targets, Geophysics, 72(2), G1-G8, 2007.

Huang, N. E. and Wu, Z.: A review on Hilbert-Huang transform: the method and its applications on geophysical studies, Rev. Geophys., 46, RG 2006, doi:10.1029/2007RG000228, 2008.

Huang, N. E., Shen, Z., Long, S. R., Wu, M. C., Shih, H.-H., Zheng, Q., Yen, N.-C., Tung, C.-C., and Liu, H.-H.: The empirical mode 
decomposition and the Hilbert spectrum for nonlinear and nonstationary time series analysis, P. R. Soc. London A, 454, 903995, 1998.

Irving, J. D. and Knight, R. J.: Removal of wavelet dispersion from ground-penetrating radar data, Geophysics, 68(3), 960970, 2003.

Jackson, L. P. and Mound, J. E.: Geomagnetic variation on decadal time scales: What can we learn from Empirical Mode Decomposition?, Geophys. Res. Lett., 37, L14307, doi:10.1029/2010GL043455, 2010.

Jeffryes, P.: A method of seismic surveying with overlapping shot times, US Patent 7,050,356, 2002.

Jeng, Y., Li, Y.-W., Chen, C.-S., and Chien, H.-Y.: Adaptive filtering of random noise in near-surface seismic and ground-penetrating radar data, J. Appl. Geophys., 68, 36-46, doi:10.1016/j.jappgeo.2008.08.013, 2009.

Jeng, Y., Lin, M.-J., Chen, C.-S., and Wang, Y.-H.: Noise reduction and data recovery for a very low frequency electromagnetic survey using the nonlinear decomposition method, Geophysics, 72(5), F223-F235, 2007.

Lee, T. and Ouarda, T. B. M. J.: Long term prediction of precipitation and hydrologic extremes with nonstationary oscillation processes, J. Geophys. Res., 115, D13107, doi:10.1029/2009JD012801, 2010.

Lin, M.-J. and Jeng, Y.: Application of the VLF-EM method with EEMD to the study of a mud volcano in southern Taiwan, Geomorphology, 119(1-2), 97-110, doi:10.1016/j.geomorph.2010.02.021, 2010.
Meunier, J. and Bianchi, T.: Harmonic noise reduction opens the way for array size reduction in vibroseis operations, 72nd Annual International Meeting, SEG, Expanded Abstr., 70-73, 2002.

Nyman, D. C. and Gaiser, J. E.: Adaptive rejection of highline contamination: 53rd Annual International Meeting, SEG, Expanded Abstr., 321-323, 1983.

Peel, M. C. and McMahon, T. A.: Recent frequency component changes in interannual climate variability, Geophys. Res. Lett., 33, L16810, doi:10.1029/2006GL025670, 2006.

Saucier, A., Marchant, M., and Chouteau, M.: A fast and accurate frequency estimation method for canceling harmonic noise in geophysical records, Geophysics, 71(1), V7-V18, 2006.

Tsai, H.-R.: The velocity structures of gas hydrate from wide-angle seismic tomography in the offshore SW Taiwan, M.S. thesis, Institute of Applied Geosciences, National Taiwan Ocean University, Taiwan, ROC, 96 pp., 2007.

Tsolis, G. S. and Xenos, T. D.: Seismo-ionospheric coupling correlation analysis of earthquakes in Greece, using empirical mode decomposition, Nonlin. Processes Geophys., 16, 123-130, doi:10.5194/npg-16-123-2009, 2009.

Wu, Z. and Huang, N. E.: Ensemble empirical mode decomposition: A noise-assisted data analysis method, COLA Tech. Rep. 193, Cent. for Ocean-Land-Atmos. Stud., Calverton, Md., 2005.

Wu, Z. and Huang, N. E.: Ensemble empirical mode decomposition: A noise-assisted data analysis method, Advances in Adaptive Data Analysis, 1(1), 1-41, 2009. 